\title{
The Neurocognitive Cost of Enhancing Cognition with Methylphenidate: Improved Distractor Resistance but Impaired Updating
}

\author{
Sean James Fallon ${ }^{1,3}$, Marieke E. van der Schaaf ${ }^{1,2}$, Niels ter Huurne ${ }^{2}$, and Roshan Cools Re $^{1,2}$
}

\begin{abstract}
A balance has to be struck between supporting distractorresistant representations in working memory and allowing those representations to be updated. Catecholamine, particularly dopamine, transmission has been proposed to modulate the balance between the stability and flexibility of working memory representations. However, it is unclear whether drugs that increase catecholamine transmission, such as methylphenidate, optimize this balance in a task-dependent manner or bias the system toward stability at the expense of flexibility (or vice versa). Here we demonstrate, using pharmacological fMRI, that methylphenidate improves the ability to resist distraction (cognitive stability) but
\end{abstract}

\section{INTRODUCTION}

Methylphenidate is used to treat attention deficit hyperactivity disorder (ADHD; Swanson et al., 1998) and is also taken by healthy adults in the hope of improving academic and cognitive performance (Maher, 2008). Methylphenidate acts by blocking the dopamine transporter (Gatley et al., 1999; Ritz, Lamb, Goldberg, \& Kuhar, 1987), leading to increased dopamine and noradrenaline in pFC (Berridge et al., 2006) and increased dopamine in striatal areas (Volkow et al., 2001).

Despite the widespread use of methylphenidate, characterizing its precise cognitive effects has proved to be a difficult task. For example, despite the well-known dependence of working memory on catecholaminergic tone (Berridge \& Arnsten, 2013; Vijayraghavan, Wang, Birnbaum, Williams, \& Arnsten, 2007; Zahrt, Taylor, Mathew, \& Arnsten, 1997; Brozoski, Brown, Rosvold, \& Goldman, 1979), the effects of methylphenidate on working memory are variable (Ilieva, Hook, \& Farah, 2015; Smith \& Farah, 2011). Some of this variability likely reflects individual differences in dopamine release (Clatworthy et al., 2009). However, the precise nature of the task demands is now also known to contribute to the direction

\footnotetext{
${ }^{1}$ Radboud University Donders Institute of Brain, Cognition, and Behavior, ${ }^{2}$ Radboud University Nijmegen Medical Centre, ${ }^{3}$ University of Oxford

impairs the ability to flexibly update items currently held in working memory (cognitive flexibility). These behavioral effects were accompanied by task-general effects in the striatum and opposite and task-specific effects on neural signal in the pFC. This suggests that methylphenidate exerts its cognitive enhancing and impairing effects through acting on the pFC, an effect likely associated with methylphenidate's action on the striatum. These findings highlight that methylphenidate acts as a double-edged sword, improving one cognitive function at the expense of another, while also elucidating the neurocognitive mechanisms underlying these paradoxical effects.

and extent of catecholaminergic drug effects (Fallon et al., 2015; Linssen, Sambeth, Vuurman, \& Riedel, 2014; Bari \& Robbins, 2013; Floresco, 2013; Mehta, Manes, Magnolfi, Sahakian, \& Robbins, 2004; Cools, Barker, Sahakian, \& Robbins, 2001; Gotham, Brown, \& Marsden, 1988).

Although much research on working memory focuses on delay-related stabilization of memoranda, it is widely recognized that attentional mechanisms - the gating and selection of specific stimuli-play an integral part in determining the life span and vivacity of remembered items (Fallon, Zokaei, \& Husain, 2016; Gazzaley \& Nobre, 2012). A balance has to be struck between supporting robust mental representations that are resistant to interference (cognitive stability) and allowing those representations to be sufficiently flexible to be updated (cognitive flexibility; Braver \& Cohen, 2000). Thus, although it may be advantageous in certain contexts to keep hold of information and protect it from distraction, it is also important to efficiently update information that is no longer relevant. However, the extent to which gating the entry of items into working memory is affected by changes in catecholaminergic tone and the neural locus of these effects have not been examined. According to existing neurocomputational models, methylphenidate, through modulating dopamine levels, might have antagonistic effects on the stability versus flexibility of mnemonic representations (Durstewitz \& Seamans, 2008; Hazy, Frank, \& O'Reilly, 2007), an idea that runs counter to its clinical 
usage, and the suggestion that methylphenidate acts as a uniform cognitive enhancer.

The neural locus of any differential effect on stability versus flexibility is hotly contested. Dopamine has been hypothesized to modulate the balance between cognitive stability and flexibility through acting on the pFC (Durstewitz \& Seamans, 2008), the striatum (Hazy et al., 2007), or through there being a division of labor, with the frontal cortex promoting stability and the striatum promoting flexibility (Cools \& D'Esposito, 2011). In particular the dorsal striatum, and its modulation by dopamine, has been implicated in updating (or preparing to update) items in working memory (Fallon \& Cools, 2014; Yu, FitzGerald, \& Friston, 2013; Murty et al., 2011; Dahlin, Neely, Larsson, Backman, \& Nyberg, 2008; Hazy et al., 2007). This study sought to adjudicate between these hypotheses by examining whether any effect of methylphenidate on the tradeoff between cognitive stability and flexibility is accompanied by neural changes in the frontal cortex and/or dorsal striatum using pharmacological fMRI.

Although methylphenidate is assumed to act on cognitive functions directly, there is another possibility: Methylphenidate may exert its effects on working memory vicariously, via boosting the response to rewarding events that subsequently alter the mnemonic functions of the $\mathrm{pFC}$. This hypothesis concurs generally with recent observations that working memory allocation is valuebased (Chatham \& Badre, 2013; Dayan, 2012; Dixon \& Christoff, 2012; Hazy et al., 2007). More directly, previously we have found that the reward-related BOLD signal increase in the ventral striatum for an unexpected financial gain, compared with a loss, predicted its effect on ignoring or updating items in working memory (Fallon \& Cools, 2014). Given that reward-related processing in the ventral striatum is thought to be dopaminergic in origin (Knutson \& Gibbs, 2007), methylphenidate might modify the ventral striatal response to gains versus losses, which in turn exerts downstream effects on cognitive control. Thus, we also assessed the hypothesis that methylphenidate affects cognitive control vicariously through modulating ventral striatal reward response.

Using pharmacological fMRI, we examined the effect of methylphenidate on a modified delay match-to-sample task in a double-blind placebo-controlled crossover study. The task was modified to include two additional phases during the delay period between encoding and probe (Figure 1). First, participants received an unexpected outcome (gain, neutral, or loss) after playing a gamble. Next they were presented with novel intervening stimuli, which had to be either ignored or updated in working memory.

\section{METHODS}

\section{Participants}

Twenty-four participants (11 men, 13 women) gave written informed consent to take part in this study and were compensated for participation ( $€ 130)$. Three participants were excluded (two due to incomplete data due to scanner malfunction and one due to inability to perform task in the scanner; mean accuracy $\sim 38 \%$ ). The remaining participants were aged between 19 and 28 years (mean $=21.40$ years $)$.

\section{Inclusion and Exclusion Criteria}

Exclusion criteria for participation in the study were as follows: uncorrected visual impairment (e.g., color blindness), history of neurological or psychiatric disorder,

Figure 1. An illustration of the modified delay match-to-sample task used (Fallon \& Cools, 2014). Participants were presented with two stimuli that always had to be encoded (2000 msec). After a variable delay period (2000-6000 msec), a $1500-$ msec response screen requested participants to make a choice to receive a gain, neutral, or loss outcome (1000 msec). After another variable delay period (2000$6000 \mathrm{msec}$ ), participants were presented with novel stimuli (1000 msec) that had to be ignored or used for working memory updating (displacing

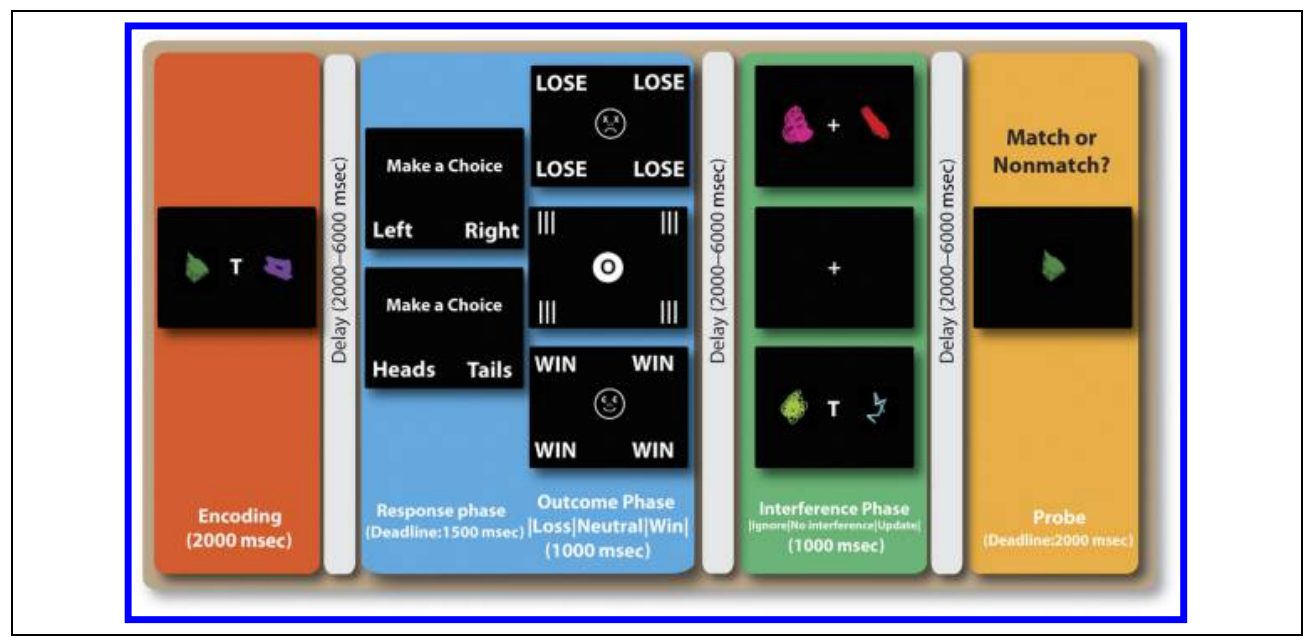
the original targets). A no-interference condition, in which only a fixation cross appeared (1000 msec), was included as a control condition. After another variable delay period (2000-6000 msec), participants were presented with a probe item. Participants had to responding according to whether the presented item matched or did not match one of those items in the same. In the present example, the correct answer would be a match if the current trial was in the ignore or no-interference condition, but a nonmatch in the update condition. They had a maximum of 2000 msec to respond. 
history of medical treatment to head or neck, history of asthma, history of disorder that may affect metabolism or circulation (diabetes, hypertension, cardiac arrhythmia), currently taking over-the-counter medications, pregnancy, metallic implants, current smoker, consumption of $>20$ units alcohol or 20 cigarettes per week, history of hard drug use (e.g., heroin or cocaine), cannabis use $<2$ weeks before testing and history, left-handedness, tattoos, unremovable body piercings, claustrophobia, and frequent gambling (e.g., casino, fruit machines).

\section{Intake and Testing Procedures}

Participants took part in an intake session, during which they performed some baseline tests and were trained on the tasks that they had to perform on each of the test days. Participants were screened by a medical doctor (NtH) to check for contraindications for taking methylphenidate (family history of tics, etc.) and were screened by a psychologist (MvdS) using the Mini International Neuropsychiatric Interview (Sheehan et al., 1998) to assess any history of psychiatric problems. Physiological measurements (height, weight, pulse rate, and blood pressure) and the following psychological measures were also assessed: depression (Beck Depression Inventory; Beck, Ward, Mendelson, Mock, \& Erbaugh, 1961), trait anxiety (Spielberger Trait Anxiety Inventory; Spielberger, Gorsuch, \& Lushene, 1970), and ADHD symptomatology (Pappas, 2006).

\section{Pharmacological Design and Procedure}

Participants were tested using a within-subject, doubleblind, placebo-controlled cross-over design on two separate occasions, once after taking $20 \mathrm{mg}$ methylphenidate (Ritalin) and once after taking a placebo substance, with the order counterbalanced across participants. This dose was chosen as it has been found to exert cognitive effects (Elliott et al., 1997; though see Linssen et al., 2011). The drug and placebo sessions occurred with a minimum of a 1-week gap and a maximum of 4-week gap. Scanning took place roughly $1 \mathrm{hr}$ after drug administration.

\section{Task Procedure}

The task is a modified delay match-to-sample task and has been fully described (Fallon \& Cools, 2014; Figure 1). Each trial contained four distinct phases separated by three delay periods.

\section{Encoding Phase}

Participants were presented with the letter "T" in the middle of the screen flanked by two computer-generated "spirographs" of different colors. Note that every shape was novel and had a different color (RGB value). The presence of a " $\mathrm{T}$ " in the center of the screen indicated that the stimuli had to be remembered as the "Target" stimuli.

\section{Outcome Phase}

On the gain and loss trials, participants had to guess the outcome of a coin toss by making a head or tails response with their index or middle finger, respectively. Two screens were then presented: a "LOSS" screen with a negative auditory tone ("horn"; a loss of $€ 1$ ); or a "WIN" screen with a positive auditory tone (cash register; a win of $€ 1$ ). Gains and losses did not depend on performance, were equally frequent, and did not lead to a net loss/gain. In a control condition, participants just made a left or right response, after which they saw a neutral screen.

\section{Interference Phase}

Participants were presented with two novel stimuli that had to be ignored or updated according to the absence or presence of the letter "T." Updated stimuli displaced the previous set of stimuli as the target stimuli. There was also a control condition containing only a fixation cross (no interference).

\section{Probe Phase}

A single stimulus was presented in the center of the screen. Participants made a match or nonmatch response with their middle or index finger. There was an equal probability of the probe being a target or nontarget stimulus. Half of nontarget stimuli were novel stimuli; the other half were nontargets (dumped items in the update condition or distractors in the ignore condition).

An interval of $2000 \mathrm{msec}$ separated each trial. Participant's block-wise accuracy was shown after each block. Trials on which participants had to ignore or update information during the delay period were separated into blocks to reduce task-switching effects and reduce the possibility that the drug modulated performance by increasing cognitive switching.

There were five runs in each session. Each run contained four blocks (two update and two ignore blocks, order randomized, nine trials per block). In each block, there were three gain, three loss, and three neutral outcome trials, during which they could gain, lose, or receive no money.

Our main index of behavior was accuracy. Accuracy scores were arcsine transformed to conform to parametric assumptions (Howell, 1997). This transform did not alter the significance of the result.

\section{fMRI Statistical Data Analysis}

\section{Data Acquisition}

Participants were scanned at the Donders Centre for Cognitive Neuroimaging using a Siemens Tim Trio 3-T 
Scanner. A multiecho EPI sequence was used to acquire T2 $*$-weighted images (repetition time $=2.32$, echo times $=$ 9, 19.3, 30, and $40 \mathrm{msec}$, flip angle $=90^{\circ}$ ). Each run consisted of $1360(4 \times 340$ scans per echo) scans of 38 slices (slice thickness $=2.5 \mathrm{~mm}$, resolution $=3.3 \mathrm{~mm} \times 3.3 \mathrm{~mm} \times$ $2.5 \mathrm{~mm}$ ). Thirty scans were acquired at the beginning of each testing day to use as "weighing scans" (see below). Participants were scanned using a 32-channel head coil. Movement was minimized by positioning two cushions to either side of the participant's head and by placing tape across participant's forehead. Participants completed five runs of the experimental task. A high-resolution structural scan of the brain was also acquired during the intake session (MPRAGE: 192 scans, repetition time $=2300 \mathrm{msec}$, echo time $=3.03 \mathrm{msec}$ ) to aid with preprocessing (see below).

\section{Data Preprocessing}

Data were preprocessed in SPM5 using a pipeline optimized for analyzing multiecho data (Poser, Versluis, Hoogduin, \& Norris, 2006) and was the same pipeline as used previously (Fallon \& Cools, 2014). This involved using data from the first echo to estimate the six rigid body realignment parameters $(x, y, z$, roll, pitch, yaw) needed to align the images and applying these realignment parameters to data from the other three echoes. Correction for image misalignment was performed by aligning the first image of all the subsequent runs to the first image of the first run and then realigning each image in each run to the first image in that run.

Images from the four echoes were then combined into a single image as a weighted-sum of data from the four echoes. On each testing day, 30 "weighing" scans were acquired. This enabled us to perform a voxel-specific weighing between the four echoes using in-house software, which maximized the contribution of each echo according to its contrast to noise ratio (see Poser et al., 2006). The combined images were then slice-time corrected to the middle slice, coregistered with participant's structural MPRAGE and then normalized to a standard template (Montreal Neuroimaging Institute) using the "unified segmentation" procedure (Ashburner \& Friston, 2005). The data were smoothed with an 8-mm FWHM Gaussian kernel.

\section{Comparison of Movement across Drug Sessions}

The absolute mean level of movement (mm) in the $x, y$, $z$ directions were $.10, .12$, and .36 , respectively. The mean angular movement (degrees) in the roll, pitch, and yaw directions were $.005, .004$, and .003, respectively. Furthermore, there were no significant differences between the placebo and drug sessions in the absolute level of movement on any of these six metrics (all $t$ s $<1$ ).

\section{Modeling}

\section{First-level Modeling}

The following events were modeled: initial encoding stimuli, "loss," "neutral," "win" screen, to-be-ignored stimuli, fixation cross (no interference condition), to-beupdated stimuli and probe event, and end of block accuracy screen. In addition, parametric modulators, orthogonalized to the task regressor, were entered for the three working memory conditions (ignore, no interference, and update) regressors. These regressors corresponded to the valence of the received outcome on the current trial ( 1 for loss, 2 for neutral and 3 for gain). This modeling approach (Fallon \& Cools, 2014) enabled us to examine whether the response to the intervening stimuli was modulated in a linear fashion by the valence of the preceding outcome. Finally, a parametric regressor corresponding to the RT for the probe event was added. All task-related regressors were convolved with a canonical hemodynamic response function. The first-level design matrices were adapted to deal with nuisance variables in the exact same way as our previous study (Fallon \& Cools, 2014). Following the approach of Lund, Norgaard, Rostrup, Rowe, and Paulson (2005), 24 nuisance regressors were included in the model: the six realignment parameters used to realign each image, the square of these realignment parameters, the first derivative of these realignment parameters, and the realignment parameters used to realign the previous volume (to account for spin-history effect; Friston, Williams, Howard, Frackowiak, \& Turner, 1996). A high-pass filtered (128 sec) was applied to the data to remove low frequency signals and an AR(1) model was applied to adjust for serial correlations. Microtime onsets were adjusted to take into account the earlier slice-timing correction.

\section{Second-level Modeling}

This study aimed to investigate whether methylphenidate modulates reward processing, working memory, and their interaction. Accordingly, we built three full factorial models to answer our principal questions. In all of these models, regressor-based beta weight maps were passed to the second level. Drug effects on the BOLD response to intervening stimuli were examined in a 2 (update, ignore) $\times 2$ (drug, placebo) factorial model. Similarly, drug effects on the outcome phase were examined by entering contrast images (beta weights) into a 2 (gain, loss) $\times 2$ (methylphenidate, placebo) full factorial model. Finally, task and valence interactions were examined in a 2 (parametric modulation of update response, parametric modulation of ignore response) $\times 2$ (drug, placebo) full factorial model.

For each model, main effects of task, drug, and the interaction between task and drug were evaluated using $F$ tests and followed up, where appropriate, with $t$ test to illustrate the direction of the effects. Random field theory 
Figure 2. Accuracy scores (proportion correct) for each task as a function of drug (A). BOLD signal in the left dorsolateral $\mathrm{pFC}$

(B; $x=-44, y=18$, $z=38)$ and dorsomedial $\mathrm{pFC}(\mathrm{C} ; x=-2 y=38$ $z=38$ ) was significantly modulated by drug (methylphenidate) in a task-specific (ignore vs. update) manner. Bars represent percent signal change (see Methods section for calculation) with a 3-mm sphere around the respective peak voxels for the interaction between task and drug. BOLD signal during the no-interference trials are shown for illustrative purposes. For comparison, BOLD signal in the left dorsal striatum $(x=-18 y=12 z=4,3 \mathrm{~mm}$ sphere extracted from update minus ignore) is shown (D).

In all graphs, error bars represent the standard error of the difference between methylphenidate and placebo according to each condition. DLPFC $=$ dorsolateral $\mathrm{pFC} ;$ DMPFC $=$ dorsomedial $\mathrm{pFC}$.

was used to correct the $p$ values at the voxel level for multiple comparisons within our a priori generated ROIs.

\section{ROI Selection}

This experiment was designed to address the following question: Does methylphenidate affect working memory through acting on the dorsal striatum and/or pFC? This question justified the use of an ROI approach. We generated ROIs directly based on independent data from our previous study (Fallon \& Cools, 2014), which used the same task with the same parameters. For the model examining frontostriatal BOLD signal during the interference phase, we composed an intervening stimulus-related ROI that included the striatum (defined as regions significantly activated, $p<.01$ for update minus ignore trials) and the pFC (defined as regions significantly activated, $p<.01$ for ignore minus update trials). For the outcome phase model, we generated an outcome-related ROI by comparing gain minus loss events at the outcome phase in our previous data ( $p<.01$, uncorrected). Previously we did not identify a significant Reward $\times$ Task interaction and therefore had no a priori predictions about the neural locus of this contrast's effects. Thus, it was not warranted to generate an ROI for this contrast.

\section{Data Visualization}

Regions that showed statistically significant differences between conditions (Figure 2B, C) were plotted using rfxplot (rfxplot.sourceforge.net/) and displayed as percent signal change: \% signal change $=(\operatorname{Beta}($ task $) \times$ $\max (\mathrm{HRF}) \times 100) /($ Beta $($ constant $))($ see Glascher, 2009 , for more details).

\section{RESULTS}

\section{Behavioral Data}

To compare the effect of drug on working memory as a function of task demands and outcome valence, we examined accuracy scores using a Drug (methylphenidate, placebo) $\times$ Task (ignore, no interference, update) Drug $\times$ Task $\times$ Reward (loss, neutral, or gain) repeated-measures ANOVA. Methylphenidate differentially affected accuracy as a function of task, as confirmed by a significant interaction between task and drug $(F(2,40)=20.16, p=.0000008$; Figure $2 \mathrm{~A})$. Simple main effects analysis revealed that this was due to methylphenidate, relative to placebo, improving performance on ignore trials $(F(1,20)=15.29, p=$ $.01)$ and no-interference trials $(F(1,20)=10.72, p=$ $.04)$. In contrast, methylphenidate impaired performance on update trials $(F(1,20)=8.44, p=.0088)$. Furthermore, follow-up tests revealed that methylphenidate, relative to placebo, disproportionately improved performance on ignore trials compared with no-interference trials $(F(1$, $40)=5.43, p=.03)$. There was a significant main effect of Task $\left(F(2,40)=78.63, p=1.4 \times 10^{-14}\right)$ but no significant main effect of Drug $(F(1,20)=1.83, p=.19)$ or reward $(F<1)$. None of the other interaction effects were significant $(F s<1)$. Of note, accuracy for novel 
probes was significantly higher compared with those for distracter probes in both the ignore $(t(20)=3.417, p=$ $.003)$ and update conditions $(t(20)=2.35, p=.029)$. Thus, indicating that participants processed the distracters in the ignore condition and the initial targets in the update condition. Moreover, there was no significant interaction between Drug, Task, and Probe type or between Drug and Probe type $\left(F_{\mathrm{S}}<1\right)$. Further supplementary analysis confirmed that there was no significant difference in accuracy between the no-interference trials sandwiched in the ignore blocks and those in the update blocks and that performance on these trial types did not vary as a function of drug $(F \mathrm{~S}<1)$.

The same statistical model that was used to evaluate accuracy was used to examine RT differences. This analysis revealed that there was a main effect of Task, $F(2$, $40)=15.83, p=.000004$. Pairwise comparisons revealed that participants were significantly faster to respond on update trials compared with ignore trials $(t(19)=4.67$, $p=.00017)$ and no interference trials $(t(19)=4.50, p=$ $.00024)$. There was no difference in response latencies for ignore and no interference trials $(t(19)=.10, p=.91)$.
There was no significant interaction between Drug and Task $(F(2,40)=1.588, p=.217)$. None of the other effects or interactions were significant $(F \mathrm{~S}<1)$.

\section{Neuroimaging Results}

Interference Phase

Main effect of task. Consistent with our previous study (Fallon \& Cools, 2014), the intervening stimuli elicited differential effects when they had to be ignored versus used for updating in a variety of frontal, parietal, occipital, and subcortical areas. Ignoring, relative to updating, was found to lead to bilateral increases in the dorsolateral $\mathrm{pFC}$ and posterior parietal cortex (angular gyri). In contrast, updating, relative to ignoring, was found to lead to bilateral increases in the dorsal striatum and posterior ventral areas extending from the inferior temporal gyri to the occipital cortex (Table 1; Figure 3A).

Main effect of Drug. During the intervening stimuli, significant $(p<.05$, small volume-corrected) main ( $F$ test)

Table 1. Results Table for Directional Tests on the Interference Phase ( $t$ Test)

\begin{tabular}{|c|c|c|c|c|c|}
\hline Contrast & Regions & $\begin{array}{l}\text { Number of } \\
\text { Voxels (k) }\end{array}$ & $\begin{array}{c}p(F W E, \text { Small } \\
\text { Volume })\end{array}$ & $t$ & $\begin{array}{c}\text { MNI } \\
\text { Coordinates } \\
(x y z)\end{array}$ \\
\hline \multirow[t]{3}{*}{ Methylphenidate minus Placebo } & Dorsal caudate nucleus & 23 & .005 & 4.4 & -16014 \\
\hline & Left putamen & 3 & .011 & 4.18 & $-242-10$ \\
\hline & Left putamen & 29 & .039 & 3.76 & -20168 \\
\hline Placebo minus Methylphenidate & Nothing & & & & \\
\hline \multirow[t]{6}{*}{ Ignore minus Update } & Left angular gyrus & 557 & $<.0001$ & 8.47 & $-58-6030$ \\
\hline & Left angular gyrus & 74 & $<.0001$ & 7.90 & $-40-7442$ \\
\hline & Left superior medial gyrus & 62 & $<.0001$ & 6.84 & -64238 \\
\hline & $\begin{array}{l}\text { Left middle frontal } \\
\text { gyrus }\end{array}$ & 101 & $<.0001$ & 6.22 & -341446 \\
\hline & Right middle frontal gyrus & 19 & $<.0001$ & 5.88 & 381644 \\
\hline & Left precuneus & 74 & $<.0001$ & 5.45 & $52-5238$ \\
\hline \multirow[t]{3}{*}{ Update minus Ignore } & Left putamen & 210 & $<.0001$ & 5.68 & -18124 \\
\hline & Right putamen & 13 & .046 & 3.70 & 20142 \\
\hline & Right putamen & 1 & .005 & 3.26 & 26164 \\
\hline [Methylphenidate-ignore minus & Dorsomedial $\mathrm{pFC}$ & 22 & .003 & 4.63 & -43838 \\
\hline $\begin{array}{l}\text { Placebo-ignore] vs. } \\
\text { [Placebo-update minus } \\
\text { Methylphenidate] }\end{array}$ & Left dorsolateral $\mathrm{pFC}$ & 10 & .021 & 3.96 & -422038 \\
\hline $\begin{array}{l}\text { [Placebo-ignore minus } \\
\text { Methylphenidate] vs. } \\
\text { [Methylphenidate } \\
\text { minus Placebo-update] }\end{array}$ & None & & & & \\
\hline
\end{tabular}

Correction for multiple comparisons was performed voxel-wise random field theory within the task-related ROI. 
Figure 3. (A) Task-related effects. Left: shown in cyan are voxels that were significant $(p<.001$, uncorrected) for the update minus ignore events collapsed across methylphenidate and placebo sessions. Right: shown in yellow are voxels that were significant $(p<.001$, uncorrected) for the update minus ignore events collapsed across methylphenidate and placebo sessions. (B) Taskand reward-related effects within a mask of the basal ganglia, collapsed across placebo and methylphenidate sessions. Shown in green are significant voxels $(p<.001$, uncorrected) for the gain minus loss events. Shown in cyan are voxels that were significant $(p<.001$, uncorrected) for the update minus ignore contrast. Note that we corrected for multiple comparisons for statistical inference (tables and text) but display at an uncorrected threshold to illustrate the physiogical plausibility of the effects.

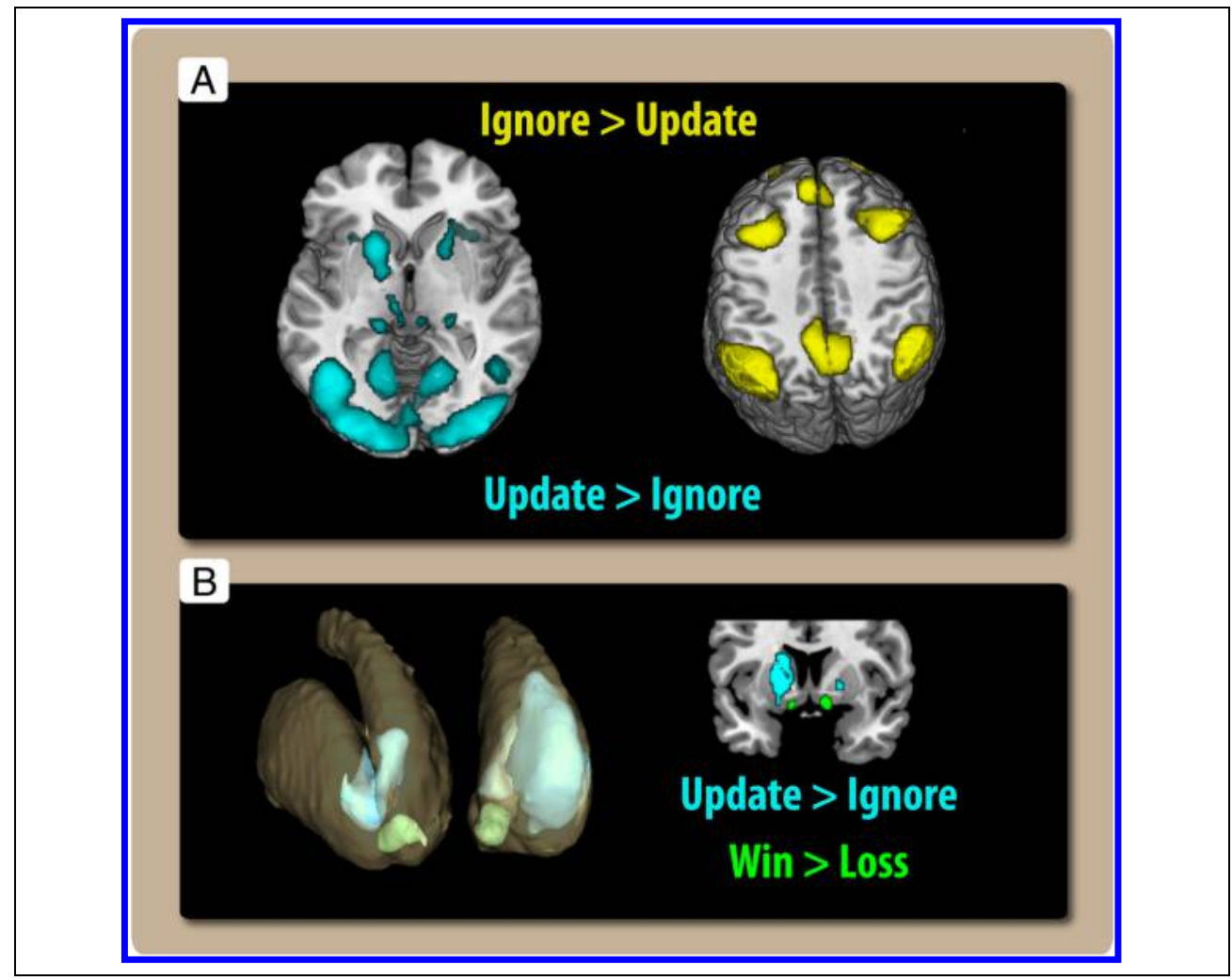

effects of Drug, irrespective of task demands, were found in the dorsal caudate nucleus $[x=-16, y=0, z=14]$ and the left putamen $[x=-24, y=2, z=-10]$. A follow-up $t$ test revealed that there was significantly increased BOLD signal in the left posterior caudate nucleus $[x=-16, y=0, z=4]$, left ventral striatum $[x=-24$, $y=2, z=-10]$, left dorsal striatum $[x=-20, y=16$, $z=8]$, right dorsal striatum $[x=24, y=18, z=2]$, and posterior ventral striatum $[x=-20, y=4, z=12]$ after methylphenidate compared with placebo (Figure 4; Table 1). There were no regions that showed significantly greater BOLD signal in placebo compared with methylphenidate (Table 1).

Drug $\times$ Task effects. In agreement with the behavioral data, there was a significant interaction between Drug and
Figure 4. Effect of methylphenidate during the interference period. Shown in purple are the areas that showed a task-general response to methylphenidate $(p<.001$, uncorrected, $F$ test for the main effects of Drug for both ignore and update trials). Shown in red are the regions that showed an interaction between Drug and Task during the interference phase $(t$ contrast between methylphenidate ignore minus placebo ignore vs. placebo update minus methylphenidate update, $p<.001$, uncorrected). Display at an uncorrected threshold was chosen for

illustrative purposes, though note that the left dorsolateral $\mathrm{pFC}$ and dorsomedial $\mathrm{pFC}$ were significant after correcting for multiple comparisons $(p<.05$, FWE, small volume-corrected) 
Task (update, ignore) in the brain (Figures 2B, C and 4). Significant effects $(p<.05$, FWE, voxel level, small volume-corrected) on BOLD signal were observed in the left dorsolateral pFC $[x=-42, y=20, z=38]$ and dorsomedial pFC $[x=-4, y=38, z=38]$.

Follow-up $t$ contrasts determined the direction of these effects. Contrasting ignore minus update trials on methylphenidate with update minus ignore trials on placebo revealed significantly $(p<.05$, FWE, voxel level, small volume-corrected) increased BOLD signal in the left dorsolateral pFC $[x=-44, y=18, z=38]$ and dorsomedial pFC $[x=-2, y=38, z=38]$ (Table 1 ). Specifically, methylphenidate, relative to placebo, increased BOLD signal in the left dorsolateral pFC on ignore trials, whereas there was no difference on update trials (Figures 2B and 4). In contrast, methylphenidate, relative to placebo, increased BOLD signal in the dorsomedial pFC during ignore trials, but decreased BOLD signal during update trials (Figures 2C and 4). The reverse contrast (placebo ignore minus methylphenidate ignore, methylphenidate update minus placebo update) did not produce any significant increases in BOLD signal.

\section{Outcome Phase}

For outcome, there were no main effects of Drug (Table 2$)$. There was a significant $(p<.05$, FWE, small volume-corrected) main effect of Outcome in the left ventral striatum $[x=-12, y=0, z=-12]$. Follow-up $t$ contrasts determined the direction of this effect. Examining gain minus loss events across both placebo and drug sessions revealed significant $(p<.05$, FWE, small volumecorrected) BOLD signal in the bilateral ventral striatum $[x=10, y=4, z=-12],[x=-12, y=0, z=-12]$ (Table 2; Figure 3B). In line with the lack of a behavioral interaction between drug and outcome, there was no significant ( $p<.05$, FWE, small volume-corrected) interaction between Methylphenidate and Outcome (Table 2).

\section{Parametric Modulation of Task by Preceding Outcome Valence}

We also investigated whether the neural response to each task condition (ignore or update) was modulated in a parametric (linear) fashion according to the valence of the preceding outcome delivery. In other words, we asked whether the BOLD response to ignore or update events increased linearly as a function of reward level. In line with the lack of interaction between Drug, Task, and Outcome at the behavioral level, there were no significant voxels ( $p<.05$, FWE whole brain) for the threeway interaction between drug, task, and outcome, that is, there were no significant voxels whose response to the task (ignore, update) varied parametrically according to the preceding valence and drug.

\section{Other Phases of the Task}

To examine whether methylphenidate also had an effect during encoding and probe, we compared the BOLD signal on methylphenidate and placebo for these two events. There was no significant $(p<.05 \mathrm{FWE})$ voxels in either the encoding or the probe phase within our task-related ROI or even at the whole-brain level.

\section{Brain-Behavioral Analyses}

Correlational analyses revealed that the methylphenidateinduced improvement in accuracy on ignore trials was related to the corresponding methylphenidate-induced increase in BOLD signal in the left dorsolateral pFC (3 $\mathrm{mm}$ sphere around the peak voxel for the interaction between Drug and Task: $x=-44, y=18, z=38$ ) during ignore trials $(\operatorname{rho}(21)=.551, p=.009$; Figure 4). However, there was no relationship between the methylphenidateinduced change in the left dorsolateral pFC during update

Table 2. Results Table for Directional Comparisons in the Outcome Phase ( $t$ Test)

\begin{tabular}{|c|c|c|c|c|c|}
\hline Contrast & Regions & $\begin{array}{l}\text { Number of } \\
\text { Voxels }(k)\end{array}$ & $\begin{array}{c}p(F W E, \text { Small } \\
\text { Volume-corrected })\end{array}$ & $t$ & $\begin{array}{c}\text { MNI Coordinates } \\
\left(\begin{array}{l}x y z \\
\end{array}\right)\end{array}$ \\
\hline \multirow[t]{2}{*}{ Win minus Loss } & Left ventral striatum & 27 & .024 & 3.98 & $\begin{array}{lll}-12 & 0 & -12\end{array}$ \\
\hline & Right ventral striatum & 37 & .035 & 3.86 & $104-12$ \\
\hline Loss minus Win & & & \multicolumn{3}{|c|}{ No regions contained within ROI } \\
\hline Methylphenidate minus Placebo & & & \multicolumn{3}{|c|}{ No regions contained within ROI } \\
\hline Placebo minus Methylphenidate & & & \multicolumn{3}{|c|}{ No regions contained within ROI } \\
\hline $\begin{array}{l}\text { Methylphenidate win minus Placebo } \\
\text { win vs. Placebo loss minus } \\
\text { Methylphenidate loss }\end{array}$ & & & \multicolumn{3}{|c|}{ No regions contained within ROI } \\
\hline $\begin{array}{l}\text { Placebo win minus Methylphenidate } \\
\text { win vs. Methylphenidate loss } \\
\text { minus Placebo loss }\end{array}$ & & & \multicolumn{3}{|c|}{ No regions contained within ROI } \\
\hline
\end{tabular}

Correction for multiple comparisons was performed voxel-wise random field theory within the task-related ROI. 
trials and change in behavioral performance $(\operatorname{rho}(21)=$ $.005, p=.984)$. There was no significant correlation between methylphenidate-induced changes in BOLD signal in the dorsomedial pFC $(3 \mathrm{~mm}$ sphere at around: $x=$ $-2, y=38, z=38$ ) and behavior for ignore trials (rho $(21)=-.159, p=.491)$ or update trials $(\operatorname{rho}(21)=$ $-.104, p=.652$ ). Similarly, there was no significant association between the effect of methylphenidate-induced changes in BOLD signal in the left dorsal striatum and ignoring $(\operatorname{rho}(21)=.190, p=.490)$ or updating $(\operatorname{rho}(21)=$ $-.240, p=.295$ ). Finally, there was no significant correlation between the effect of methylphenidate on ventral striatal BOLD response to outcome ([methylphenidate gain minus methylphenidate loss vs. placebo gain minus placebo loss]) and change in behavior in the ignore $(\operatorname{rho}(21)=.197, p=.392)$ or update conditions $(\operatorname{rho}(21)=$ $-.022, p=.92)$.

\section{DISCUSSION}

The present results demonstrate that methylphenidate improves distractor resistance at the expense updating. Thus, across-subject, drug-induced gains in ignoring interfering information were partially offset by an impaired ability to use this information to update current working memory representations.

Methylphenidate is commonly regarded as a cognitive enhancer, because it can improve certain functions (Elliott et al., 1997). In this study, however, methylphenidate did not act as a uniform cognitive enhancer. Instead, it improved cognitive stability at the expense of cognitive flexibility. Therefore, the present findings show that methylphenidate is a double-edged sword, that is, methylphenidate's beneficial cognitive effects come at a cost.

In addition to examining the differential cognitive effects of methylphenidate, this study also sought to adjudicate between competing accounts of the neural locus of these effects. The balance between cognitive stability and cognitive flexibility in working memory has been argued to be regulated by dopamine in frontal (Fallon, Williams-Gray, Barker, Owen, \& Hampshire, 2013; Durstewitz \& Seamans, 2008; Rolls, Loh, Deco, \& Winterer, 2008) and striatal brain regions (Cools \& D'Esposito, 2011; Hazy et al., 2007). Our fMRI results serve to unify seemingly contradictory and disparate reports on the neural basis of catecholamine-induced cognitive changes by showing that task-specific and task-general effects of methylphenidate are distributed between the frontal cortex and the striatum respectively. The contrasting effects of methylphenidate on working memory were associated with contrasting effects on the pFC. Specifically, methylphenidate increased BOLD signal in the left dorsolateral $\mathrm{pFC}$ and the dorsomedial $\mathrm{pFC}$ during ignore trials, while decreasing BOLD signal in the dorsomedial $\mathrm{pFC}$ during update trials (Figures $2 \mathrm{~B}-\mathrm{C}$ and 4 ). This finding concurs with prior observations that catecholamines can act directly on the pFC to elicit adaptive dynamics of cognitive control (Berridge \& Arnsten, 2015; Ott,
Jacob, \& Nieder, 2014; Floresco, 2013; Arnsten \& Dudley, 2005), for example, by modulating short-term synaptic plasticity (Stokes et al., 2013; Mongillo, Barak, \& Tsodyks, 2008; Gonzalez-Burgos, Kroener, Seamans, Lewis, \& Barrionuevo, 2005). Moreover, neurons in this region have been found to be exquisitely sensitive to the neurochemical niche they occupy (Seamans \& Yang, 2004; Williams \& Goldman-Rakic, 1995). Specifically, our effects might be captured by dualstate theory (Durstewitz \& Seamans, 2008), a recurrent network model of pFC dynamics, grounded in neurophysiology, according to which dopamine contributes critically to flexible transitions between different network states in the pFC. In this model, pFC networks can be either in a D1-dominated state, associated with intermediate levels of dopamine, which is characterized by a high-energy barrier favoring robust stabilization of representations, or in a D2dominated state, associated with (low or) high levels of dopamine, which is characterized by a low-energy barrier favoring fast, flexible shifting between representations. Here methylphenidate modulated BOLD signal in prefrontal regions, improving cognitive stability but impairing cognitive flexibility. As such, the present results are consistent with the idea that methylphenidate exerts its therapeutic cognitive effects by acting on the pFC (Spencer, Klein, \& Berridge, 2012; Berridge et al., 2006; Arnsten \& Dudley, 2005).

In addition to observing task-specific effects in the pFC, we also observed task-general effects in the striatum. Methylphenidate potentiated BOLD signal in the striatum during the intervening stimulus across all three (ignore, no interference, and update) working memory conditions. Importantly, methylphenidate did not induce these changes during any of the other task phases (encoding, outcome, or probe), suggesting that the effects were specific to the processing of novel, intervening salient stimuli or events, irrespective of their visual features or cognitive requirements, that is, the BOLD response for to-be-ignored stimuli, to-be-updated stimuli, and fixation crosses were all enhanced after methylphenidate administration. This is consistent with current ideas about striatal dopamine's role in the processing of salience (Redgrave \& Gurney, 2006) and in registering changes in the environment that may require changes in attentional selection (van Schouwenburg, den Ouden, \& Cools, 2010). It is possible that the changes we observed in the striatum could have been a necessary precondition for effects at the pFC to arise. Indeed, methylphenidate's therapeutic and cognitive effects have repeatedly been shown to be a function of the degree to which methylphenidate increases striatal dopamine release (Clatworthy et al., 2009; Volkow, Wang, Fowler, \& Ding, 2005). In keeping with this striatal facilitation hypothesis, there is a rich set of reciprocal interactions between frontal and striatal areas, with the timbre of this relationship strongly influenced by dopamine levels in the respective areas. For example, impaired functioning of the frontal cortex and/or prefrontal dopamine depletion are known to lead to changes in striatal dopamine transmission (Meyer-Lindenberg et al., 
2002, 2005; Roberts et al., 1994; Louilot, Le Moal, \& Simon, 1989; Pycock, Kerwin, \& Carter, 1980). Conversely, changes in striatal dopamine levels and dopamine D1 receptor function have been found to alter cortical dynamics (Cole et al., 2013; Dang, O’Neil, \& Jagust, 2012; Braskie et al., 2011; Rieckmann, Karlsson, Fischer, \& Backman, 2011; Kellendonk et al., 2006). Thus, frontal responses, and their behavioral consequences, can vary according to striatal dopamine transmission. On the basis of these findings, one might hypothesize that the methylphenidate-induced changes in the striatum played a causal role in promoting the changes we observed in the frontal cortex. Of course, the current data do not provide empirical evidence for this speculation, and future work is required to test directly this alternative striatal hypothesis.

The effects of methylphenidate on working memory and associated neural responses did not vary with the valence of the preceding outcome, although this paradigm successfully elicited reward-related increases in ventral striatal BOLD signal. Given the association between methylphenidate and motivation, such a modulation by stimulants may only occur when gains or losses are contingent upon performance, that is, when the potential financial gains/losses are contingent upon performance of certain task (Wardle, Treadway, \& de Wit, 2012). There is clearly a pressing need for future studies to investigate this question. As such, the possibility that methylphenidate acts vicariously to boost cognitive function through enhancing motivation remains open.

There are a number of caveats and issues in need of explanation. One might argue that the differential effect of methylphenidate on ignoring and updating occurred because the updating task was easier than the ignoring task. For example, in the ignore condition, information had to be maintained for a longer period than the update task. Several aspects of the present result make it extremely unlikely that differential demands for maintenance underlie our drug-induced effects at the behavioral or neural level. First, with regard to behavior, the maintenance periods for the ignore and no-interference conditions were identical. Despite this, methylphenidate, compared with placebo, had differential effects on accuracy in the ignore and no-interference conditions, indicating that methylphenidate acted on the specific process of distractor resistance. Moreover, the accuracy rates for the ignore and no-interference conditions were identical for the placebo session, arguing against the idea that there was any baseline difference in intrinsic difficulty. With regards to the neural data, it should be noted that, at the point at which new information (or fixation cross) was being presented to participants, the maintenance periods for the preceding stimuli in each of the three conditions (ignore, no interference, and update) were the same. Thus, none of the neural data on drug-induced effects at the interference phase could have been affected by differences in the maintenance period. Moreover, methylphenidate had opposite effects on prefrontal BOLD signal during ignoring versus updating (Figure $2 \mathrm{~B}-\mathrm{C}$ ). Thus, despite all of the preceding experimental events and durations being identical up until the point of the interference phase, differences in drug-induced neural signaling were observed between the conditions at the very first point were the conditions started to diverge in their psychological requirements. However, further work is necessary to fully characterize the role of difficulty (or mental effort) in the effects of methylphenidate on ignoring and updating. Specifically, future experiments should vary the set size of the number of items that need to be maintained, ignored, and updated. This would be a most elegant way to disentangle task difficulty and intrinsic differences associated with having to ignore versus update information in working memory.

Our hypotheses are grounded in computational and theoretical work on the dopamine system. However, we cannot or do not claim an exclusive, or even decisive, role for dopamine in generating the present findings. For example, methylphenidate is known to affect noradrenaline levels in the pFC (Berridge et al., 2006). Therefore, it is possible that methylphenidate's effects on frontal signaling observed in this study are mediated by noradrenaline. As is the case for dopamine, optimal levels of noradrenergic stimulation are necessary for working memory performance and dynamic cognitive control (Arnsten, 2011; Aston-Jones \& Cohen, 2005). Thus, further studies are needed to determine the pharmacological specificity of these effects both with regard to both the neurotransmitters as well as the receptor subtypes.

This study has important implications for the use of methylphenidate as a cognitive enhancer in normal healthy individuals. Methylphenidate is commonly used as a treatment for $\mathrm{ADHD}$, but a growing proportion of healthy individuals are taking this drug to improve cognitive performance (Greely, 2013). The present results suggest that methylphenidate does not act as a "smart pill": Taking methylphenidate does not make an individual smarter. Rather, in agreement with its known neurochemical effects, that is, boosting catecholamine levels, methylphenidate's enhancement of a cognitive function comes at the cost of impairing other cognitive functions.

\section{Acknowledgments}

This work was conducted in the context of a VIDI and subsequently a VICI grant from the Innovational Research Incentives Scheme of the Netherlands Organisation for Scientific Research (NWO) to R. C.; a Human Frontiers Science Program grant to Kae Nakamura, Nathaniel Daw, and R. C.; and a James McDonnell Scholar Award to R. C. R. C. is a consultant to Abbott Laboratories, but she is not an employee or a stock shareholder.

Reprint requests should be sent to Sean James Fallon, Department of Experimental Psychology, University of Oxford, OX13UD, United Kingdom, or via e-mail: sean.fallon@psy.ox.ac.uk.

\section{REFERENCES}

Arnsten, A. F. (2011). Catecholamine influences on dorsolateral prefrontal cortical networks. Biological Psychiatry, 69, e89-e99. 
Arnsten, A. F., \& Dudley, A. G. (2005). Methylphenidate improves prefrontal cortical cognitive function through alpha2 adrenoceptor and dopamine D1 receptor actions: Relevance to therapeutic effects in attention deficit hyperactivity disorder. Behavioral and Brain Functions, 1, 2.

Ashburner, J., \& Friston, K. J. (2005). Unified segmentation. Neuroimage, 26, 839-851.

Aston-Jones, G., \& Cohen, J. D. (2005). An integrative theory of locus coeruleus-norepinephrine function: Adaptive gain and optimal performance. Annual Review of Neuroscience. 28, 403-450.

Bari, A., \& Robbins, T. W. (2013). Noradrenergic versus dopaminergic modulation of impulsivity, attention and monitoring behaviour in rats performing the stop-signal task: Possible relevance to ADHD. Psvchopharmacology 230, 89-111.

Beck, A., Ward, C., Mendelson, M., Mock, J., \& Erbaugh, J. (1961). An inventory for measuring depression. Archives of General Psychiatry, 4, 561-632.

Berridge, C. W., \& Arnsten, A. F. (2013). Psychostimulants and motivated behavior: Arousal and cognition. Neuroscience and Biobehavioral Reviews. 37, 1976-1984.

Berridge, C. W., \& Arnsten, A. F. (2015). Catecholamine mechanisms in the prefrontal cortex: Proven strategies for enhancing higher cognitive function. Current Opinion in Behavioral Sciences, 4, 33-40.

Berridge, C. W., Devilbiss, D. M., Andrzejewski, M. E., Arnsten, A. F., Kelley, A. E., Schmeichel, B., et al. (2006).

Methylphenidate preferentially increases catecholamine neurotransmission within the prefrontal cortex at low doses that enhance cognitive function. Biological Psychiatry, 60, 1111-1120.

Braskie, M. N., Landau, S. M., Wilcox, C. E., Taylor, S. D., O'Neil, J. P., Baker, S. L., et al. (2011). Correlations of striatal dopamine synthesis with default network deactivations during working memory in younger adults. Human Brain Mapping, 32, 947-961.

Braver, T. S., \& Cohen, J. D. (2000). On the control of control: The role of dopamine in regulating prefrontal function and working memory. In S. Mansell \& J. Driver (Eds.), Control of cognitive processes: Attention and performance XVIII (pp. 713-737). Cambridge, MA: MIT Press.

Brozoski, T. J., Brown, R. M., Rosvold, H. E., \& Goldman, P. S. (1979). Cognitive deficit caused by regional depletion of dopamine in prefrontal cortex of rhesus monkey. Science. 205, 929-932.

Chatham, C. H., \& Badre, D. (2013). Working memory management and predicted utility. Frontiers in Behavioral Neuroscience, 7, 83.

Clatworthy, P. L., Lewis, S. J., Brichard, L., Hong, Y. T., Izquierdo, D., Clark, L., et al. (2009). Dopamine release in dissociable striatal subregions predicts the different effects of oral methylphenidate on reversal learning and spatial working memory. Journal of Neuroscience, 29, 4690-4696.

Cole, D. M., Oei, N. Y., Soeter, R. P., Both, S., van Gerven, J. M., Rombouts, S. A., et al. (2013). Dopamine-dependent architecture of cortico-subcortical network connectivity. Cerebral Cortex, 23, 1509-1516.

Cools, R., Barker, R. A., Sahakian, B. J., \& Robbins, T. W. (2001). Enhanced or impaired cognitive function in Parkinson's disease as a function of dopaminergic medication and task demands. Cerebral Cortex, 11, 1136-1143.

Cools, R., \& D'Esposito, M. (2011). Inverted-U-shaped dopamine actions on human working memory and cognitive control. Biological Psychiatry, 69, e113-e125.

Dahlin, E., Neely, A. S., Larsson, A., Backman, L., \& Nyberg, L. (2008). Transfer of learning after updating training mediated by the striatum. Science, 320, 1510-1512.
Dang, L. C., O’Neil, J. P., \& Jagust, W. J. (2012). Dopamine supports coupling of attention-related networks. Lournal of Neuroscience, 32, 9582-9587.

Dayan, P. (2012). How to set the switches on this thing. Current Otinion in Neurobiology, 22, 1068-1074.

Dixon, M. L., \& Christoff, K. (2012). The decision to engage cognitive control is driven by expected reward-value: Neural and behavioral evidence. PLOS ONE, 7, e51637.

Durstewitz, D., \& Seamans, J. K. (2008). The dual-state theory of prefrontal cortex dopamine function with relevance to catechol-o-methyltransferase genotypes and schizophrenia. Biological Psvchiatrv, 64, 739-749.

Elliott, R., Sahakian, B. J., Matthews, K., Bannerjea, A., Rimmer, J., \& Robbins, T. W. (1997). Effects of methylphenidate on spatial working memory and planning in healthy young adults. Psychopharmacology, 131, 196-206.

Fallon, S. J., \& Cools, R. (2014). Reward acts on the pFC to enhance distractor resistance of working memory representations. Iournal of Cognitive Neuroscience, 26, 2812-2826.

Fallon, S. J., Smulders, K., Esselink, R. A., van de Warrenburg, B. P., Bloem, B. R., \& Cools, R. (2015). Differential optimal dopamine levels for set-shifting and working memory in Parkinson's disease. Neuropsvchologia. 77, 42-51.

Fallon, S. J., Williams-Gray, C. H., Barker, R. A., Owen, A. M., \& Hampshire, A. (2013). Prefrontal dopamine levels determine the balance between cognitive stability and flexibility. Cerebral Cortex, 23, 361-369.

Fallon, S. J., Zokaei, N., \& Husain, M. (2016). Causes and consequences of limitations in visual working memory. Annals of the New York Academv of Sciences, 1369, 40-54.

Floresco, S. B. (2013). Prefrontal dopamine and behavioral flexibility: Shifting from an "inverted-U" toward a family of functions. Frontiers in Neuroscience, 7, 62

Friston, K. J., Williams, S., Howard, R., Frackowiak, R. S., \& Turner, R. (1996). Movement-related effects in fMRI time-series. Magnetic Resonance in Medicine, 35, 346-355.

Gatley, S. J., Volkow, N. D., Gifford, A. N., Fowler, J. S., Dewey, S. L., Ding, Y. S., et al. (1999). Dopamine-transporter occupancy after intravenous doses of cocaine and methylphenidate in mice and humans. Psvchopharmacology 146, 93-100.

Gazzaley, A., \& Nobre, A. C. (2012). Top-down modulation: Bridging selective attention and working memory. Trends in Cognitive Sciences, 16, 129-135.

Glascher, J. (2009). Visualization of group inference data in functional neuroimaging. Neuroinformatics, 7, 73-82.

Gonzalez-Burgos, G., Kroener, S., Seamans, J. K., Lewis, D. A., \& Barrionuevo, G. (2005). Dopaminergic modulation of short-term synaptic plasticity in fast-spiking interneurons of primate dorsolateral prefrontal cortex. Journal of Neuropbvsiology, 94, 4168-4177.

Gotham, A. M., Brown, R. G., \& Marsden, C. D. (1988). 'Frontal' cognitive function in patients with Parkinson's disease 'on' and 'off' levodopa. Brain: A Journal of Neurology, 111, 299-321.

Greely, H. T. (2013). Some first steps toward responsible use of cognitive-enhancing drugs by the healthy. The American Iournal of Bioethics: $A / O B$, 13, 39-41.

Hazy, T. E., Frank, M. J., \& O'Reilly, R. C. (2007). Towards an executive without a homunculus: Computational models of the prefrontal cortex/basal ganglia system. Philosophical Transactions of the Royal Society of London, Series B: Biological Sciences, 362, 1601-1613.

Howell, D. C. (2012). Statistical methods for psychology. Cengage Leaning.

Ilieva, I. P., Hook, C. J., \& Farah, M. J. (2015). Prescription stimulants' effects on healthy inhibitory control, working memory, and episodic memory: A meta-analysis. Journal of Cognitive Neuroscience, 27, 1069-1089. 
Kellendonk, C., Simpson, E. H., Polan, H. J., Malleret, G., Vronskaya, S., Winiger, V., et al. (2006). Transient and selective overexpression of dopamine D2 receptors in the striatum causes persistent abnormalities in prefrontal cortex functioning. Neuron, 49, 603-615.

Knutson, B., \& Gibbs, S. E. (2007). Linking nucleus accumbens dopamine and blood oxygenation. Psychopharmacologv, 191, 813-822.

Linssen, A. M., Sambeth, A., Vuurman, E. F., \& Riedel, W. J. (2014). Cognitive effects of methylphenidate and levodopa in healthy volunteers. European Neuropsychopharmacology 24, 200-206.

Linssen, A. M., Vuurman, E. F., Sambeth, A., Nave, S., Spooren, W., Vargas, G., et al. (2011). Contingent negative variation as a dopaminergic biomarker: Evidence from dose-related effects of methylphenidate. Psychopharmacology, 218, 533-542.

Louilot, A., Le Moal, M., \& Simon, H. (1989). Opposite influences of dopaminergic pathways to the prefrontal cortex or the septum on the dopaminergic transmission in the nucleus accumbens. An in vivo voltammetric study. Neuroscience, 29, 45-56.

Lund, T. E., Norgaard, M. D., Rostrup, E., Rowe, J. B., \& Paulson, O. B. (2005). Motion or activity: Their role in intra and intersubject variation in fMRI. Neuroimage, 26, 960-964.

Maher, B. (2008). Poll results: Look who's doping. Nature, 452, 674-675.

Mehta, M. A., Manes, F. F., Magnolfi, G., Sahakian, B. J., \& Robbins, T. W. (2004). Impaired set-shifting and dissociable effects on tests of spatial working memory following the dopamine D2 receptor antagonist sulpiride in human volunteers. Psvchopharmacologv, 176, 331-342.

Meyer-Lindenberg, A., Kohn, P. D., Kolachana, B., Kippenhan, S., McInerney-Leo, A., Nussbaum, R., et al. (2005). Midbrain dopamine and prefrontal function in humans: Interaction and modulation by COMT genotype. Nature Neuroscience, 8, 594-596.

Meyer-Lindenberg, A., Miletich, R. S., Kohn, P. D., Esposito, G., Carson, R. E., Quarantelli, M., et al. (2002). Reduced prefrontal activity predicts exaggerated striatal dopaminergic function in schizophrenia. Nature Neuroscience, 5, 267-271.

Mongillo, G., Barak, O., \& Tsodyks, M. (2008). Synaptic theory of working memory. Science, 319, 1543-1546.

Murty, V. P., Sambataro, F., Radulescu, E., Altamura, M., Iudicello, J., Zoltick, B., et al. (2011). Selective updating of working memory content modulates meso-cortico-striatal activity. Neuroimage, 57, 1264-1272.

Ott, T., Jacob, S. N., \& Nieder, A. (2014). Dopamine receptors differentially enhance rule coding in primate prefrontal cortex neurons. Neuron, 84, 1317-1328.

Pappas, D. (2006). ADHD Rating Scale-IV: Checlists, Norms, and Clinical Interpretation. Journal of Psvchoeducational Assessment, 24, 172-178.

Poser, B. A., Versluis, M. J., Hoogduin, J. M., \& Norris, D. G. (2006). BOLD contrast sensitivity enhancement and artifact reduction with multiecho EPI: Parallel-acquired inhomogeneity-desensitized fMRI. Magnetic Resonance in Medicine, 55, 1227-1235.

Pycock, C. J., Kerwin, R. W., \& Carter, C. J. (1980). Effect of lesion of cortical dopamine terminals on subcortical dopamine receptors in rats. Nature, 286, 74-76.

Redgrave, P., \& Gurney, K. (2006). The short-latency dopamine signal: A role in discovering novel actions? Nature Reviews. Neuroscience, 7, 967-975.

Rieckmann, A., Karlsson, S., Fischer, H., \& Backman, L. (2011). Caudate dopamine D1 receptor density is associated with individual differences in frontoparietal connectivity during working memory. Journal of Neuroscience, 31, 14284-14290.
Ritz, M. C., Lamb, R. J., Goldberg, S. R., \& Kuhar, M. J. (1987). Cocaine receptors on dopamine transporters are related to self-administration of cocaine. Science, 237, 1219-1223.

Roberts, A. C., De Salvia, M. A., Wilkinson, L. S., Collins, P., Muir, J. L., Everitt, B. J., et al. (1994). 6-Hydroxydopamine lesions of the prefrontal cortex in monkeys enhance performance on an analog of the Wisconsin Card Sort Test: Possible interactions with subcortical dopamine. Journal of Neuroscience, 14, 2531-2544.

Rolls, E. T., Loh, M., Deco, G., \& Winterer, G. (2008). Computational models of schizophrenia and dopamine modulation in the prefrontal cortex. Nature Reviews. Neuroscience, 9, 696-709.

Seamans, J. K., \& Yang, C. R. (2004). The principal features and mechanisms of dopamine modulation in the prefrontal cortex. Progress in Neurobiologv, 74, 1-58.

Sheehan, D. V., Lecrubier, Y., Sheehan, K. H., Amorim, P., Janavs, J., Weiller, E., et al. (1998). The Mini-International Neuropsychiatric Interview (MINI): The development and validation of a structured diagnostic psychiatric interview for DSM-IV and ICD-10. Journal of Clinical Psychiatry, 59(Suppl. 20), 22-33.

Smith, M. E., \& Farah, M. J. (2011). Are prescription stimulants "smart pills"? The epidemiology and cognitive neuroscience of prescription stimulant use by normal healthy individuals. Psychological Bulletin, 137, 717-741.

Spencer, R. C., Klein, R. M., \& Berridge, C. W. (2012). Psychostimulants act within the prefrontal cortex to improve cognitive function. Biological Psychiatry, 72, 221-227.

Spielberger, G., Gorsuch, R. L., \& Lushene, R. E. (1970). Manual for the State-Trait Anxiety Inventory. Palo Alto, CA: Consulting Psychologists Press.

Stokes, M. G., Kusunoki, M., Sigala, N., Nili, H., Gaffan, D., \& Duncan, J. (2013). Dynamic coding for cognitive control in prefrontal cortex. Neuron, 78, 364-375.

Swanson, J. M., Sergeant, J. A., Taylor, E., Sonuga-Barke, E. J., Jensen, P. S., \& Cantwell, D. P. (1998). Attention-deficit hyperactivity disorder and hyperkinetic disorder. Lancet. 351, 429-433.

van Schouwenburg, M. R., den Ouden, H. E., \& Cools, R. (2010). The human basal ganglia modulate frontal-posterior connectivity during attention shifting. Journal of Neuroscience, 30, 9910-9918.

Vijayraghavan, S., Wang, M., Birnbaum, S. G., Williams, G. V., \& Arnsten, A. F. (2007). Inverted-U dopamine D1 receptor actions on prefrontal neurons engaged in working memory. Nature Neuroscience, 10, 376-384.

Volkow, N. D., Wang, G., Fowler, J. S., Logan, J., Gerasimov, M., Maynard, L., et al. (2001). Therapeutic doses of oral methylphenidate significantly increase extracellular dopamine in the human brain. Journal of Neuroscience, 21, RC121.

Volkow, N. D., Wang, G. J., Fowler, J. S., \& Ding, Y. S. (2005). Imaging the effects of methylphenidate on brain dopamine: New model on its therapeutic actions for attention-deficit/ hyperactivity disorder. Biological Psvchiatry , 57, 1410-1415.

Wardle, M. C., Treadway, M. T., \& de Wit, H. (2012). Caffeine increases psychomotor performance on the effort expenditure for rewards task. Pharmacologv. Biochemistrv. and Behavior, 102, 526-531.

Williams, G. V., \& Goldman-Rakic, P. S. (1995). Modulation of memory fields by dopamine D1 receptors in prefrontal cortex. Nature, 376, 572-575.

Yu, Y., FitzGerald, T. H., \& Friston, K. J. (2013). Working memory and anticipatory set modulate midbrain and putamen activity. Journal of Neuroscience, 33, 14040-14047.

Zahrt, J., Taylor, J. R., Mathew, R. G., \& Arnsten, A. F. (1997). Supranormal stimulation of D1 dopamine receptors in the rodent prefrontal cortex impairs spatial working memory performance. Journal of Neuroscience, 17, 8528-8535. 


\section{This article has been cited by:}

1. James M. Broadway, Michael J. Frank, James F. Cavanagh. 2018. Dopamine D2 agonist affects visuospatial working memory distractor interference depending on individual differences in baseline working memory span. Cognitive, Affective, \& Bebavioral Neuroscience 18:3, 509-520. [Crossref]

2. Yoav Kessler. 2017. The Role of Working Memory Gating in Task Switching: A Procedural Version of the Reference-Back Paradigm. Frontiers in Psychology 8. . [Crossref]

3. Anne E. Manktelow, David K. Menon, Barbara J. Sahakian, Emmanuel A. Stamatakis. 2017. Working Memory after Traumatic Brain Injury: The Neural Basis of Improved Performance with Methylphenidate. Frontiers in Bebavioral Neuroscience 11. . [Crossref]

4. Sean James Fallon, Nahid Zokaei, Agnes Norbury, Sanjay G. Manohar, Masud Husain. 2017. Dopamine Alters the Fidelity of Working Memory Representations according to Attentional Demands. Journal of Cognitive Neuroscience 29:4, 728-738. [Abstract] [Full Text] [PDF] [PDF Plus] 